\title{
Effect of Extended $\pi$-Conjugation of Central Cores on Photovoltaic Properties of Asymmetric Wide-Bandgap Nonfullerene Acceptors
}

\author{
Tian Xia ${ }^{\mathrm{a}}$ \\ Chao $\mathbf{L i}^{\mathrm{a}}$ \\ Hwa Sook Ryu ${ }^{b}$ \\ Xiaobo Sun ${ }^{a}$ \\ Han Young Woo*b (i) \\ Yanming Sun*a ${ }^{(B)}$ \\ a School of Chemistry, Beihang University, Beijing 100191,China \\ b Department of Chemistry, College of Science, Korea University, Seoul 136-713, \\ Republic of Korea \\ hywoo@korea.ac.kr; sunym@buaa.edu.cn
}

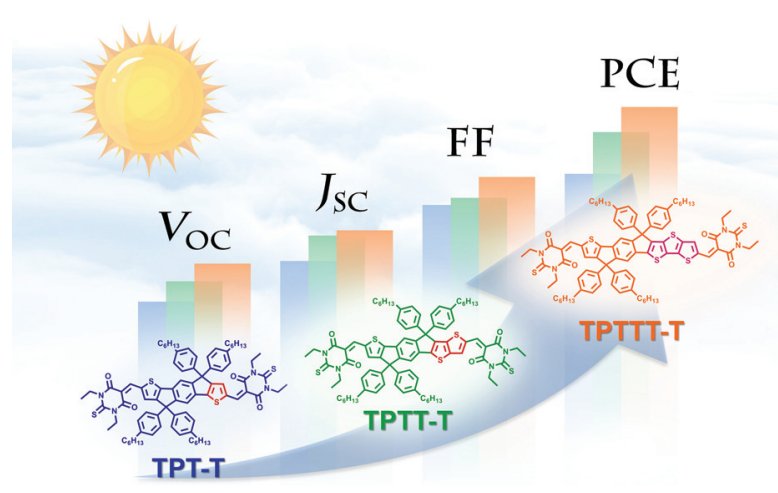

Tuning core conjugation in the wide-bandgap acceptors
Received: 2.02.2020

Accepted after revision: 12.03 .2020

DOI: 10.1055/s-0040-1709999; Art ID: 200003oa

License terms: (c)

(c) 2019. The Author(s). This is an open access article published by Thieme under the terms of the Creative Commons Attribution-NonDerivative-NonCommercial-License, permitting copying and reproduction so long as the original work is given appropriate credit. Contents may not be used for commercial purposes, or adapted, remixed, transformed or built upon. (https://creativecommons.org/licenses/by-nc-nd/4.0/).

Abstract Tremendous efforts have been dedicated to develop lowbandgap nonfullerene acceptors (NFAs) for organic solar cells (OSCs). In comparison, the NFAs with a wide bandgap (WBG) have received less attention. Here, three novel acceptor-donor-acceptor type WBG NFAs (TPT-T, TPTT-T, and TPTTT-T) have been designed and synthesized with different fused central cores by varying the $\pi$-conjugation length and molecular structures, which all exhibit wide optical bandgaps over $1.8 \mathrm{eV}$. The successive $\pi$-conjugation extension of electron-donating central cores (from TPT-T to TPTT-T and to TPTTT-T) induces red-shifted absorption spectra, lower optical bandgap, and enhanced electron mobility simultaneously. As a result, the optimized OSC devices based on TPTTT-T:PBT1-C achieved a power conversion efficiency of $7.42 \%$, which was higher than those of PBT1-C:TPTT-T (6.93\%) and PBT1-C:TPT$\mathrm{T}(6.11 \%)$ based devices. These results indicate that extending the molecular $\pi$-conjugation structure is an effective strategy to design efficient NFAs for OSCs.

Key words organic solar cells, nonfullerene acceptors, $\pi$-conjugation, wide-bandgap, power conversion efficiency

\section{Introduction}

Bulk-heterojunction organic solar cells (OSCs) have gained significant progress owing to their irreplaceable advantages, such as light weight, semitransparency, solution processability, and mechanical flexibility for roll-to-roll large-area printing manufacturing and wearable devices. ${ }^{1-7}$ Generally, the photoactive layer of OSCs consists of p-type polymer donor and n-type semiconductor acceptor materials. In the past few decades, n-type acceptors have been nearly dominated by fullerene derivatives owing to their high electron mobility and excellent isotropic chargetransport abilities. ${ }^{8-12}$ Nevertheless, the drawbacks of the fullerene acceptors, including high production costs, weak absorption of the visible light, and limited tunability of molecular energy levels, severely restrict their further applications in OSCs. ${ }^{13-15}$ Therefore, the small molecular nonfullerene acceptors (NFAs), possessing significant superiorities of wider and stronger absorption and adjustable energy levels, have gradually become promising alternatives to fullerene derivatives. ${ }^{16-23}$

Recently, the NFAs with an acceptor-donor-acceptor (A$\mathrm{D}-\mathrm{A})$ structure have attracted great attention. ${ }^{24-35}$ Through the rational chemical modification of either a $D$ or A unit, the absorption spectra, molecular energy levels, intramolecular charge transfer(ICT) effect, and intermolecular interactions of the NFAs can be effectively modulated. Up to now, a library of low-bandgap (LBG) A-D-A-type NFAs has been designed and synthesized, which have effectively promoted the development of efficient OSCs. ${ }^{24-26,36-56}$ However, few studies have focused on wide-bandgap (WBG; $>1.80 \mathrm{eV}$ ) A-D-A-type NFAs. In fact, it is quite important to explore WBG accepters, since they can generate relatively higher open-circuit voltage $\left(V_{\text {oc }}\right)$ for photovoltaic devices, which is beneficial for direct driving portable consumer electronic devices. ${ }^{57}$ Besides, it would facilitate optimization of the tandem and ternary OSCs owing to the complementary absorption combined with numerous LBG NFAs. ${ }^{58-62}$

Among the various structural engineering methods for molecular design, reducing the ICT effect by combining weakly electron-donating and electron-withdrawing units 
is one of the effective strategies to design WBG A-D-A-type NFAs. ${ }^{63}$ For example, Liu et al. reported a WBG A-D-A-type NFA IDTT-T based on indacenodithienothiophene (IDTT) core, with weakly electron-deficient barbituric acid as the terminal A unit. When paired with an LBG polymer donor, PTB7-Th, a 10\% power conversion efficiency (PCE) with a high $V_{\text {oc }}$ of $1.01 \mathrm{~V}$ was achieved. ${ }^{57}$ Huang and coworkers designed four WBG A-D-A-type NFAs and studied the effects of four different terminal groups on the device performances systematically. ${ }^{63}$ Recently, Zhu and colleagues reported a WBG A-D-A-type NFA NIDBT based on a novel ladder-type heteroarene central core, indacenodibenzothiophene (IDBT), which has strong aromaticity and weak electron-donating ability. ${ }^{64}$ However, the effect of $\pi$ conjugation extension in central cores on the photovoltaic performance of WBG NFAs has rarely been studied. Meanwhile, the development of the asymmetric NFAs has shown great potential for improving the performance of nonfullerene OSCs. ${ }^{65}$ Therefore, it should be interesting to design a novel WBG acceptor by combining the extended $\pi$ conjugation structure and an asymmetric core strategy.

In this work, three WBG NFAs were developed based on A-D-A-type structures with different fused central cores by varying the $\pi$-conjugation length and molecular structures (from a symmetric to an asymmetric structure). The molecular structures of the NFAs are shown in Scheme 1. Compared with symmetric TPT-T, the central cores of asymmetric TPTT-T and TPTTT-T had one and two more thiophene units fused on one side of central cores, respectively. The extended conjugated structures led to red-shifted absorption spectra, elevated LUMO levels, and higher electron mobilities of the NFAs, which gave rise to different PCEs of $6.11 \%, 6.93 \%$, and $7.42 \%$ for the OSCs based on TPT-T, TPTT-T, and TPTTT-T with a polymer donor (PBT1C; Figure S1), respectively. A detailed investigation, including charge transport, exciton dissociation, and charge collection properties, morphology and molecular orientation, and packing behavior, was performed to understand

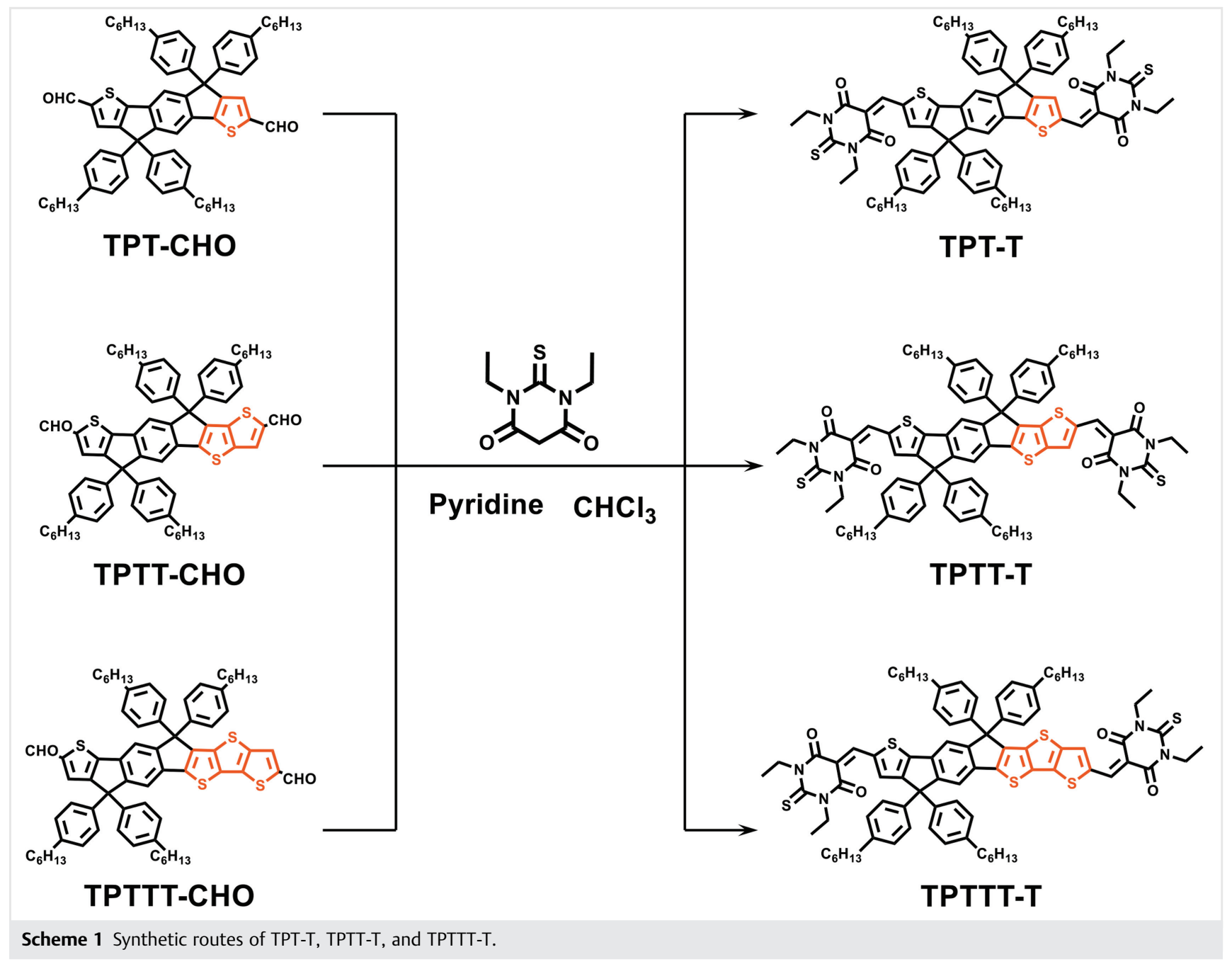


the effect of asymmetric extension of a conjugated core on the photovoltaic properties of a new series of WBG NFAs.

\section{Results and Discussion}

\section{Material Synthesis and Characterization}

The molecular structures of three WBG NFAs(TPT-T, TPTT-T, and TPTTT-T) are shown in Scheme 1. The compound TPT-T has a symmetric molecular structure. By combining one or more thiophene units with the terminal thiophene unit on the right side of the TPT-T central core, two asymmetric compounds (TPTT-T and TPTTT-T) were synthesized with extended molecular conjugated structures. The synthetic routes to TPT-T, TPTT-T, and TPTTT-T are illustrated in Scheme 1. The intermediates TPT-CHO, ${ }^{66}$ TPTT-CHO, ${ }^{30}$ and TPTTT$\mathrm{CHO}^{31}$ were prepared by following the previously reported literature. Three WBG acceptors TPT-T, TPTT-T, and TPTTT-T were prepared via the Knoevenagel condensation reaction of 1,3-diethyl-2-thioxodihydropyrimidine-4,6(1H,5H)-dione with TPT-CHO, TPTT-CHO, and TPTTT-CHO, respectively. The detailed synthetic procedures are provided in the Supporting Information and all the compounds were fully characterized by ${ }^{1} \mathrm{H}$ NMR, ${ }^{13} \mathrm{C} \mathrm{NMR}$, and matrix-assisted laser desorption ionization-time of flight (MALDI-TOF) mass spectrometry.

The normalized absorption spectra of three acceptors in dilute chloroform solutions and thin films are presented in Figure 1a,b. The corresponding data are collected in Table 1. In solution, TPT-T, TPTT-T, and TPTTT-T displayed the maximum absorption peaks at 598, 611, and $622 \mathrm{~nm}$, respectively, along with prominent shoulder peaks. But, in the solid films, all the acceptors showed broader absorption spectra compared with those in solution. Furthermore, the absorption maxima of TPT-T, TPTT-T, and TPTTT-T were redshifted to 611,622 , and $632 \mathrm{~nm}$, respectively, due to the (a)

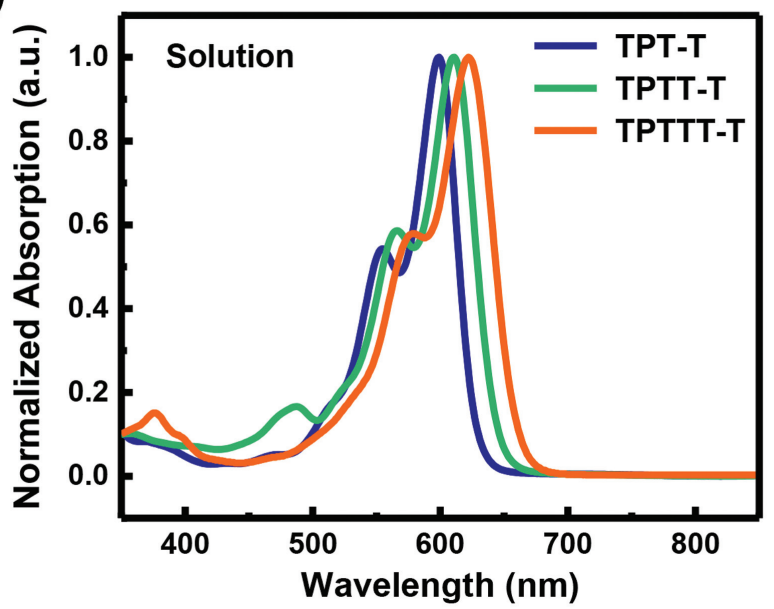

(c)

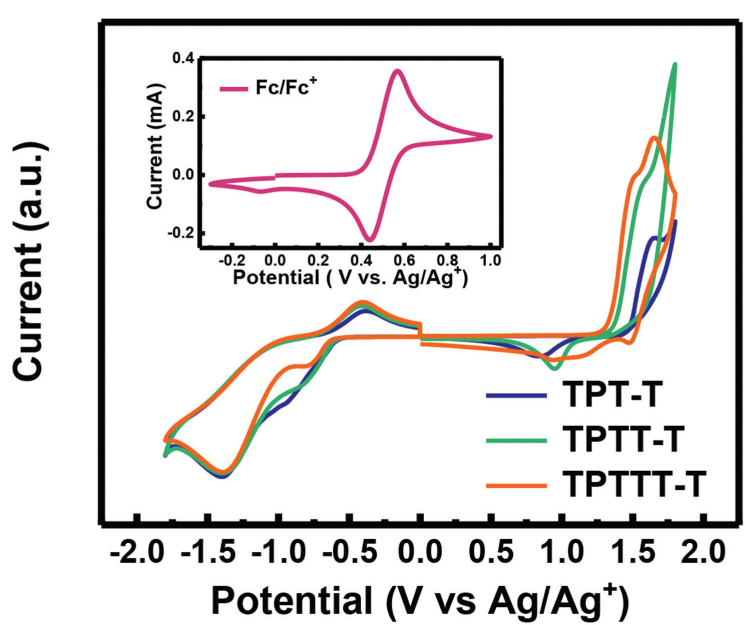

(b)

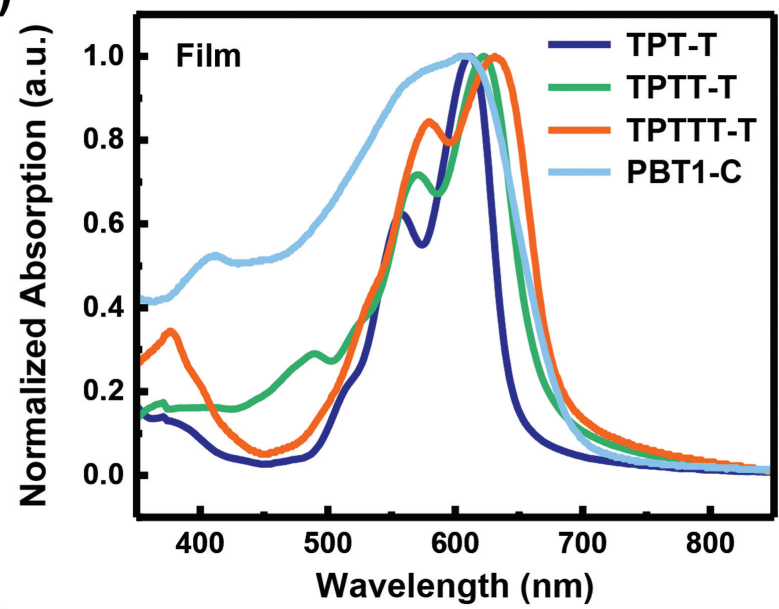

(d)

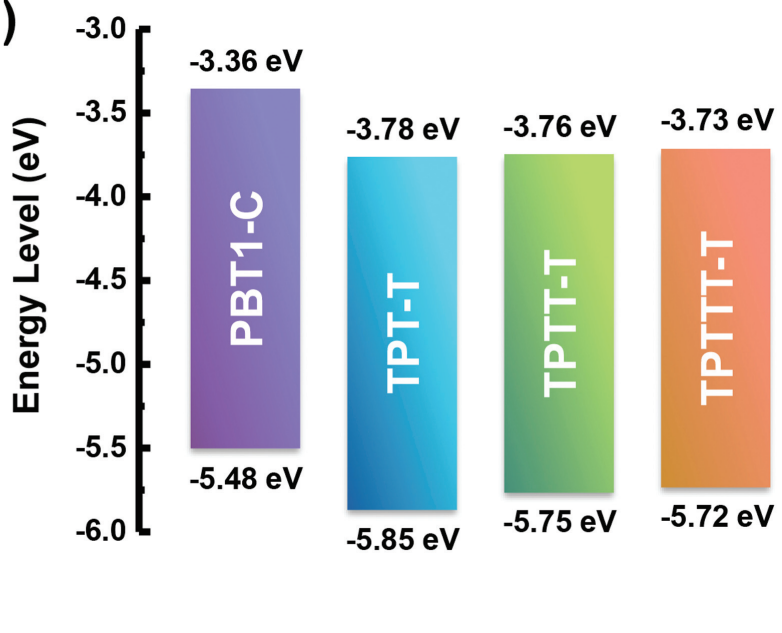

Figure 1 Normalized absorption spectra of the WBG NFAs (a) in chloroform solution and (b) in thin films. (c) Cyclic voltammograms. (d) Energy level diagram of PBT1-C and the WBG NFAs. 
Table 1 Optical and electrochemical data of the three WBG NFAs

\begin{tabular}{lllllllll}
\hline NFA & $\lambda_{\max }(\mathrm{nm})^{\mathrm{a}}$ & $\lambda_{\max }(\mathrm{nm})^{\mathrm{b}}$ & $\lambda_{\text {onset }}(\mathrm{nm})^{\mathrm{b}}$ & $E_{\mathrm{g}}{ }^{\mathrm{opt}}(\mathrm{eV})^{\mathrm{c}}$ & $E_{\mathrm{ox}}(\mathrm{V})$ & $E_{\text {red }}(\mathrm{V})$ & HOMO $(\mathrm{eV})$ & LUMO $(\mathrm{eV})$ \\
\hline TPT-T & 598 & 611 & 643 & 1.93 & 1.48 & -0.59 & -5.85 & -3.78 \\
TPTT-T & 611 & 622 & 665 & 1.86 & 1.38 & -0.61 & -5.75 & -3.76 \\
TPTTT-T & 622 & 632 & 684 & 1.81 & 1.35 & -0.64 & -5.72 & -3.73 \\
\hline
\end{tabular}

${ }^{\mathrm{a}}$ In $\mathrm{CHCl}_{3}$ solution.

b In thin film drop-casted from $\mathrm{CHCl}_{3}$ solution.

${ }^{c}$ Estimated from the empirical formula: $E_{\mathrm{g}}{ }^{\mathrm{opt}}=1240 / \lambda_{\text {onset }}$.

strong intermolecular interactions in the solid states. The onset of TPT-T, TPTT-T, and TPTTT-T films was located at $\approx 643,665$, and $684 \mathrm{~nm}$, which could be converted to the optical bandgap $\left(E_{\mathrm{g}}{ }^{\text {opt }}\right)$ of $1.93,1.86$, and $1.81 \mathrm{eV}$, respectively. Thus, these three acceptors belong to WBG semiconductor materials. From compound TPT-T to TPTTT-T, the red-shifted absorption with a narrower optical bandgap could be ascribed to the gradual extension of $\pi$-conjugation in the central cores.

Cyclic voltammetry (CV) measurements were employed to study the electrochemical properties of the NFAs. As shown in Figure 1c, the onset oxidation/reduction potentials of TPT-T, TPTT-T, and TPTTT-T (relative to $\mathrm{Ag} / \mathrm{Ag}^{+}$) were measured to be $1.48 /-0.59,1.38 /-0.61$, and $1.35 /-0.64 \mathrm{~V}$, respectively. Therefore, the HOMO/LUMO energy levels of TPT-T, TPTT-T, and TPTTT-T were determined to be $-5.85 /-3.78$, $-5.75 /-3.76$, and $-5.72 /-3.73 \mathrm{eV}$, respectively (Figure $1 \mathrm{~d}$ ). These data suggest that the extended conjugation elevates both HOMO and LUMO levels of the acceptor molecules, in which, the increase in the HOMO levels was more conspicuous than that in the LUMO levels. In addition, TPTT-T and TPTTT-T presented higher LUMO levels due to the extended conjugation and enhanced electron-donating ability of the central core, which is conducive to obtaining a higher $V_{\mathrm{oc}}$ in OSCs.

\section{Photovoltaic Properties}

To evaluate the photovoltaic characteristics of the WBG NFAs, the OSCs were fabricated with an inverted structure of ITO/ZnO/PBT1-C:NFA/ $\mathrm{MoO}_{3} / \mathrm{Ag}$. The WBG polymer donor PBT1-C was employed as a donor material. ${ }^{67}$ First, we tested the donor/acceptor $(\mathrm{D} / \mathrm{A})$ blend ratio, and the optimal D/A ratio was found to be $1: 1$ (by weight) for all of the devices. With chlorobenzene as a processing solvent, the as-cast device based on PBT1-C:TPTTT-T delivered a PCE of $6.08 \%$ with a $V_{\text {oc }}$ of $1.154 \mathrm{~V}$, a short-circuit current density $\left(J_{\mathrm{sc}}\right)$ of $9.76 \mathrm{~mA} \mathrm{~cm}^{-2}$, and fill factor (FF) of $54.0 \%$, respectively, showing superior photovoltaic performances over PBT1-C: TPT-T and PBT1-C:TPTT-T blends under the same processing conditions (as shown in Figure S2 and Table S1). Despite a rather high $V_{\mathrm{oc}}$ of over $1.1 \mathrm{~V}$, the relatively low $J_{\mathrm{sc}}$ and FF still limited the PCEs of the devices, and thermal annealing was further conducted to adjust the blend morphology. The detailed device results are exhibited in Figure S3 and Table S2. The $J-V$ curves and corresponding photovoltaic data of the optimized devices are depicted in Figure 2a and Table 2. It was found that the optimized thermal annealing temperature was $120{ }^{\circ} \mathrm{C}$ for PBT1-C:TPT-T and $140{ }^{\circ} \mathrm{C}$ for both PBT1-C:TPTT-T and PBT1-C:TPTTT-T blends. After the thermal treatment, the PBT1-C:TPTTT-T-based device yielded the highest PCE of $7.42 \%$ with a $V_{\text {oc }}$ of $1.109 \mathrm{~V}$, a $J_{\mathrm{sc}}$ of $11.06 \mathrm{~mA} \mathrm{~cm} \mathrm{~cm}^{-2}$, and a FF of $60.5 \%$, respectively. By contrast, the optimized PBT1-C:TPTT-T-based device exhibited a moderate PCE of $6.93 \%\left(V_{\mathrm{oc}}\right.$ of $1.084 \mathrm{~V}, J_{\mathrm{sc}}$ of $10.94 \mathrm{~mA} \mathrm{~cm}^{-2}$, and FF of 58.4\%), and the PBT1-C:TPT-Tbased device presented a relatively low PCE of $6.11 \%$ ( $V_{\mathrm{oc}}$ of $1.068 \mathrm{~V}, J_{\mathrm{sc}}$ of $9.85 \mathrm{~mA} \mathrm{~cm}^{-2}$, and FF of $58.1 \%$ ). These results suggest that extended $\pi$-conjugation of the NFAs could promote the photovoltaic parameters $\left(V_{\mathrm{oc}}, J_{\mathrm{sc}}\right.$, and FF) of the corresponding devices. In addition, these three WBG NFAbased devices all exhibited a high $V_{\text {oc }}$, which exceeded the $V_{\text {oc }}$ values of majority of highly efficient OSCs based on A-DA-type NFAs (Figure 2c and Table S3).

The corresponding external quantum efficiency (EQE) curves of the optimized devices are provided in Figure $2 b$. All of the devices exhibited broad photoresponses over a range from 300 to $700 \mathrm{~nm}$. The PBT1-C:TPTT-T- and PBT1-C: TPTTT-T-based devices presented integrated $J_{\text {sc }}$ values of 10.45 and $10.56 \mathrm{~mA} \mathrm{~cm}^{-2}$, which were substantially higher than that of the PBT1-C:TPT-T-based device. These data indicate that TPTTT-T- and TPTT-T-based OSCs might have more efficient photon-harvesting and charge collection properties, because of their extended conjugation as well as asymmetric structures. ${ }^{65}$ Besides, these integrated $J_{\mathrm{sc}}$ values agreed well with the $J_{\mathrm{sc}}$ values extracted from $J-V$ curves, within $5 \%$ mismatch.

\section{Mobility and Charge Transfer}

The charge transport characteristics of the three pristine NFAs and their blends were investigated via space-charge limited current (SCLC) measurements. As shown in Figure S4 and Table S4, the pristine TPT-T, TPTT-T, and TPTTT-T films exhibited electron mobilities $\left(\mu_{\mathrm{e}}\right)$ of $1.65 \times 10^{-4}, 1.93 \times 10^{-4}$, and $2.60 \times 10^{-4} \mathrm{~cm}^{2} \mathrm{~V}^{-1} \mathrm{~s}^{-1}$, respectively, which indicated that the extension of the central core conjugation was beneficial to enhance the 
(a)

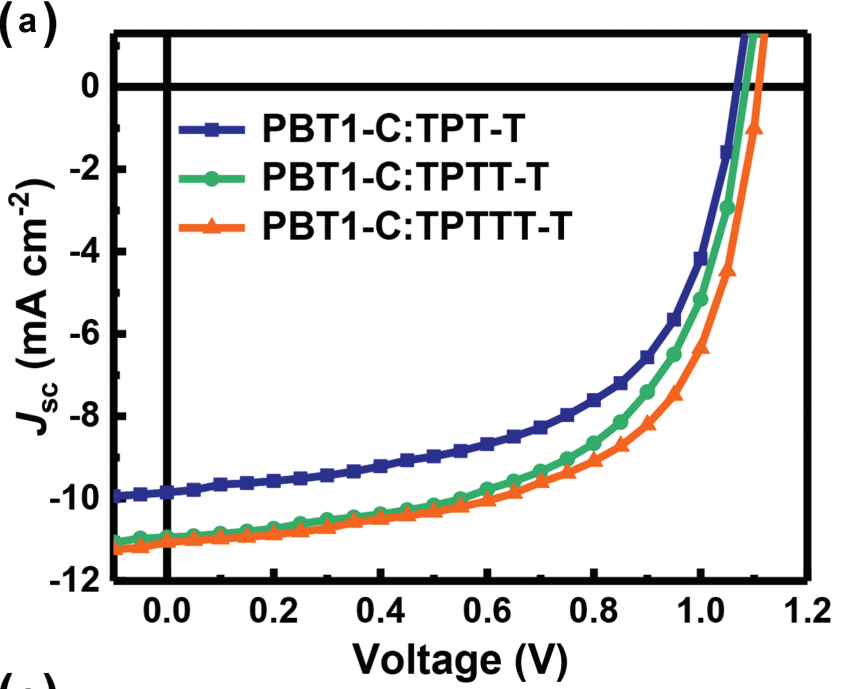

(c)

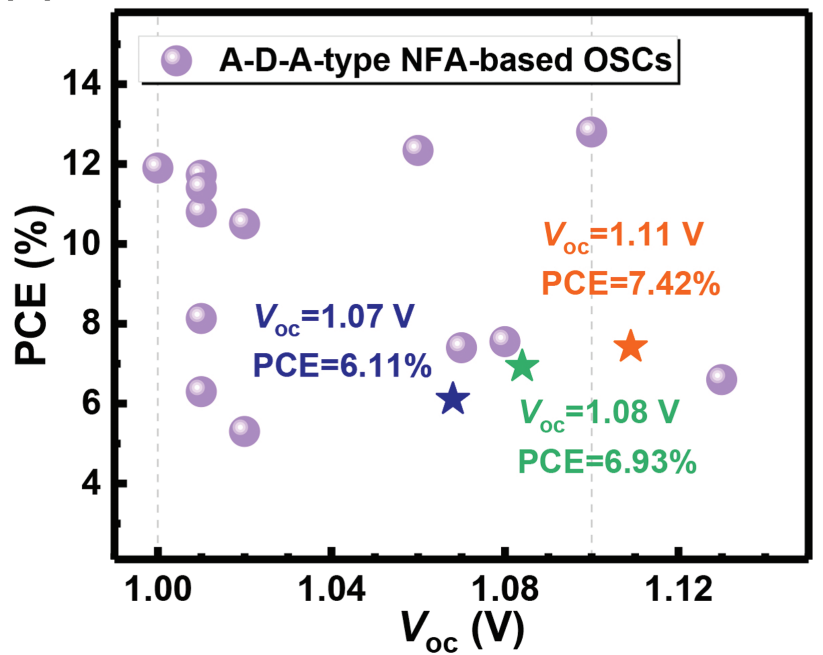

(b)

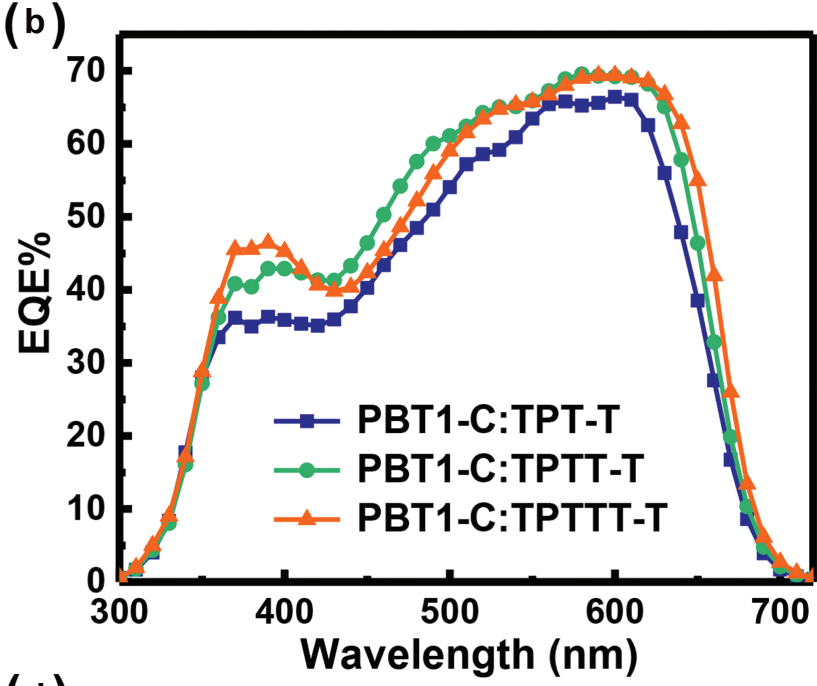

(d)

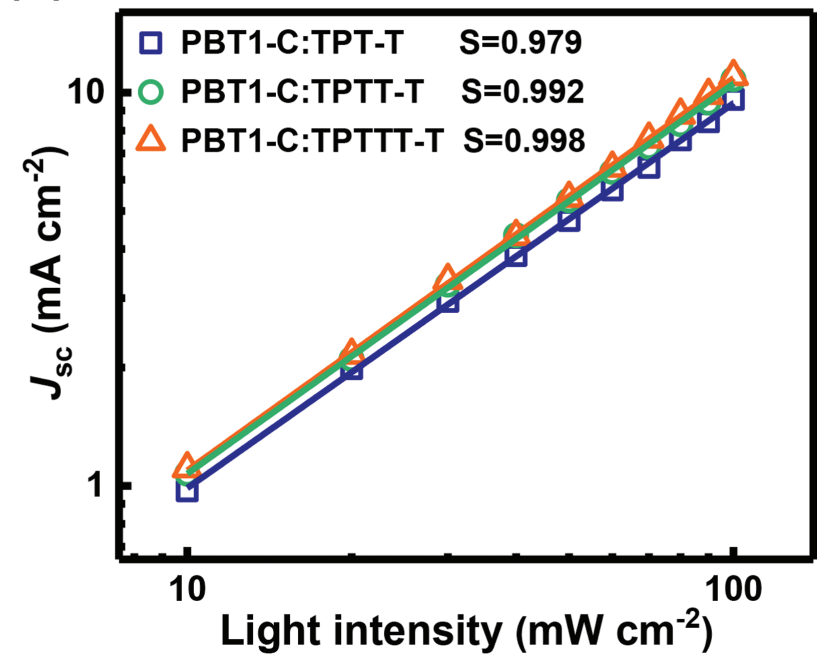

Figure 2 (a) J-V and (b) EQE curves of the optimized OSCs based on PBT1-C:NFA blends. (c) PCE against $V_{\text {oc }}$ plots of the reported A-D-A-type NFA-based organic solar cells. The list of the data is available in the Supporting Information. (d) Light-intensity-dependent sc $_{\text {c }}$ characteristics for the optimized OSCs.

Table 2 Photovoltaic parameters of the optimized OSCs based on PBT1-C:NFAs

\begin{tabular}{llllll}
\hline NFA & $V_{\text {oc }}(\mathrm{V})^{\mathrm{a}}$ & $J_{\mathrm{sc}}\left(\mathrm{mA} / \mathrm{cm}^{2}\right)^{\mathrm{a}}$ & $J_{\mathrm{sc}, \mathrm{cal}}\left(\mathrm{mA} / \mathrm{cm}^{2}\right)$ & FF $(\%)^{\mathrm{a}}$ & PCE $(\%)^{\mathrm{a}}$ \\
\hline TPT-T & $1.068(1.073 \pm 0.010)$ & $9.85(9.70 \pm 0.15)$ & 9.41 & $58.1(58.38 \pm 0.34)$ & $6.11(6.08 \pm 0.11)$ \\
TPTT-T & $1.084(1.081 \pm 0.003)$ & $10.94(11.00 \pm 0.07)$ & 10.45 & $58.4(57.03 \pm 1.11)$ & $6.93(6.78 \pm 0.13)$ \\
TPTTT-T & $1.109(1.109 \pm 0.001)$ & $11.06(11.04 \pm 0.10)$ & 10.56 & $60.5(59.28 \pm 0.90)$ & $7.42(7.26 \pm 0.13)$ \\
\hline
\end{tabular}

${ }^{\mathrm{a}}$ Average values with standard deviations were obtained from 20 devices.

electron mobility. When blending with the polymer PBT1-C, the average hole mobilities $\left(\mu_{\mathrm{h}}\right) / \mu_{\mathrm{e}}$ values of the PBT1-C: TPT-T, PBT1-C:TPTT-T, and PBT1-C:TPTTT-T devices were measured to be $20.04 \times 10^{-4} / 1.14 \times 10^{-4}, 19.58 \times 10^{-4} /$ $1.27 \times 10^{-4}$, and $19.01 \times 10^{-4} / 2.20 \times 10^{-4} \mathrm{~cm}^{2} \mathrm{~V}^{-1} \mathrm{~s}^{-1}$, with the $\mu_{\mathrm{h}} / \mu_{\mathrm{e}}$ ratios of $17.58,15.42$, and 8.64 , respectively.
It is worth noting that the PBT1-C:TPTTT-T blend films presented the highest $\mu_{\mathrm{e}}$ value with the most well-balanced $\mu_{\mathrm{h}} / \mu_{\mathrm{e}}$ ratio, which may lead to the higher $J_{\mathrm{sc}}$ and FF values of the corresponding devices.

The exciton dissociation and charge collection characteristics were studied by measuring the photocurrent 
density $\left(J_{\mathrm{ph}}\right)$ versus effective voltage $\left(V_{\mathrm{eff}}\right)$. As exhibited in Figure S5, the saturated $J_{\mathrm{ph}}\left(J_{\text {sat }}\right)$ values of the NFA-based devices could be reached at a high $V_{\text {eff }}(>2 \mathrm{~V})$, indicating that all the photogenerated charge carriers are collected effectively with negligible recombination in this region. ${ }^{68}$ The $J_{\text {sat }}$ values of the PBT1-C:TPTT-T and PBT1-C:TPTTT-T devices were measured to be 11.89 and $11.82 \mathrm{~mA} \mathrm{~cm}^{-2}$, respectively, which were higher than that of the PBT1-C: TPT-T device (11.21 $\mathrm{mA} \mathrm{cm}^{-2}$ ). The higher $J_{\mathrm{ph}}$ values via the extension of the conjugated core improved the resulting $J_{\mathrm{sc}}$ values of OSCs. Under the short-circuit conditions, the exciton dissociation probability $\left(P_{\text {diss }}=J_{\mathrm{ph}} / J_{\text {sat }}\right)$ values of the PBT1-C:TPT-T, PBT1-C:TPTT-T, and PBT1-C:TPTTT-T devices were calculated to be $88.1 \%, 91.3 \%$, and $93.3 \%$, respectively, which suggested that the devices based on asymmetric acceptors with extended conjugated cores had more efficient exciton dissociation and charge collection efficiency. ${ }^{65}$ As a result, the optimal device of PBT1-C: TPTTT-T showed the best $J_{\mathrm{sc}}$ and FF values among the three devices.

Furthermore, the charge recombination behavior was investigated by measuring the evolutionary trend of the $J_{\mathrm{sc}}$ depending on light intensity $\left(P_{\text {light }}\right)$. The relationship between the $J_{\text {sc }}$ and $P$ can be interpreted by the exponential formula: $J_{\mathrm{sc}} \infty P_{\text {light }}$, wherein the exponential factor $s$ represents the degree of bimolecular recombination. ${ }^{69}$ As displayed in Figure 2d, the $s$ values of the TPT-T-, TPTT-T-, and TPTTT-T-based devices were determined to be 0.979 , 0.992 , and 0.998 , respectively. These data imply that the TPTT-T- and TPTTT-T-based devices show a relatively weaker bimolecular recombination compared with the TPT-T-based device, which also agrees well with the higher $J_{\text {sc }}$ and FF values of the TPTT-T- and TPTTT-T based OSCs.

\section{Morphology}

To get a deeper insight into the morphologies of these NFA-based active layers in OSCs, the atomic force microscopy (AFM) measurements were performed. Figure 3 displays smooth and uniform surfaces in height images of the blend films with a rather small root mean square roughness of $0.859 \mathrm{~nm}$ for PBT1-C:TPT-T, $0.873 \mathrm{~nm}$ for PBT1-C:TPTT-T, and $0.866 \mathrm{~nm}$ for PBT1-C:TPTTT-T blend films. In addition, all the blends present a fibril network morphology in the AFM phase images, and the donor/acceptor phases are well intermixed, which is responsible for the efficient exciton dissociation and charge carrier transport. ${ }^{7}$

In addition, 2D grazing incident wide-angle X-ray scattering (GIWAXS) measurements were performed to understand the molecular orientation and packing behaviors of these acceptors in the pristine and blend films. The 2D GIWAXS patterns and the corresponding line-cuts are illustrated in Figure 4. It can be seen that all the neat films of
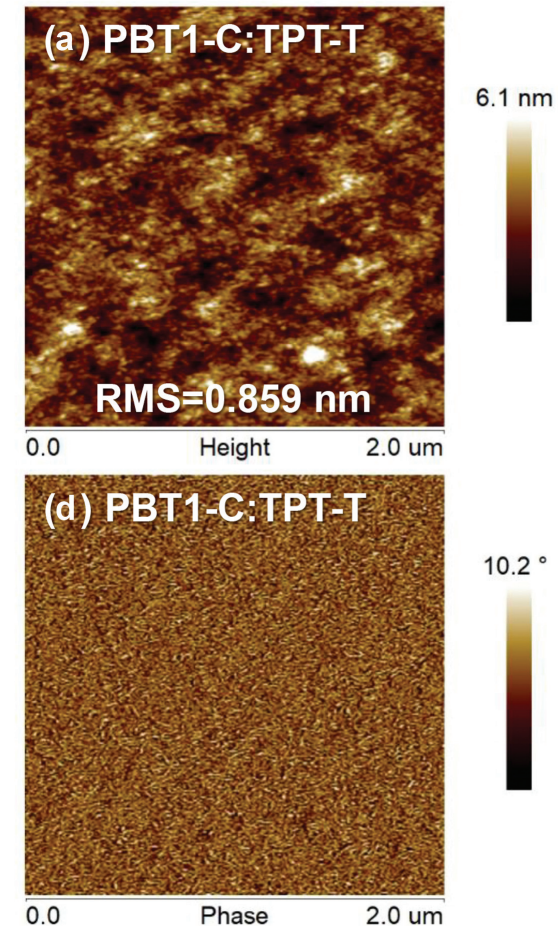
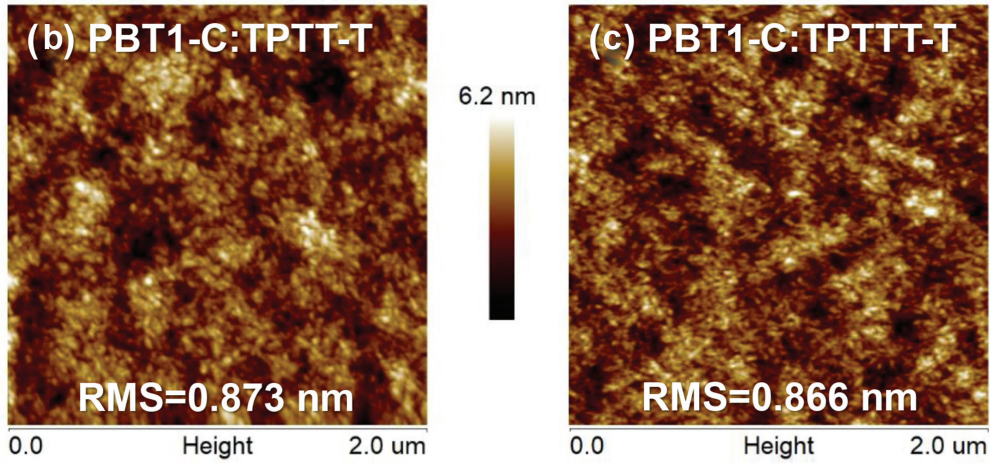

$6.0 \mathrm{~nm}$
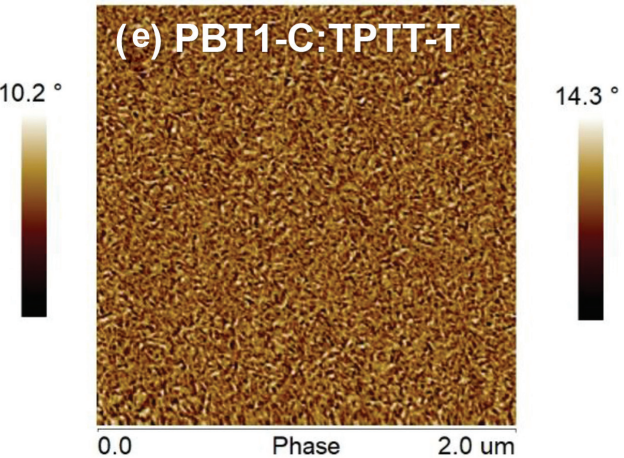

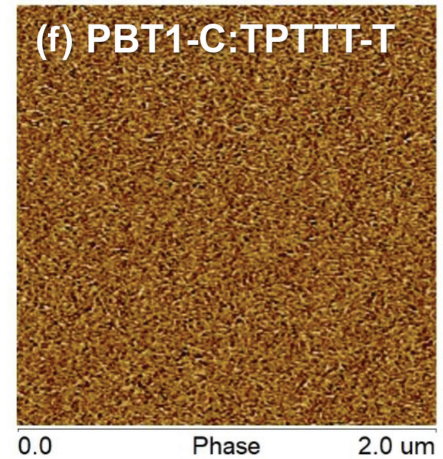

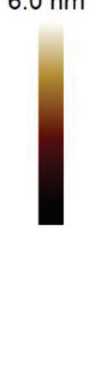

$15.1^{\circ}$
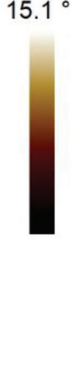

Figure 3 (a-c) AFM height images and (d-f) AFM phase images of the blend films. 

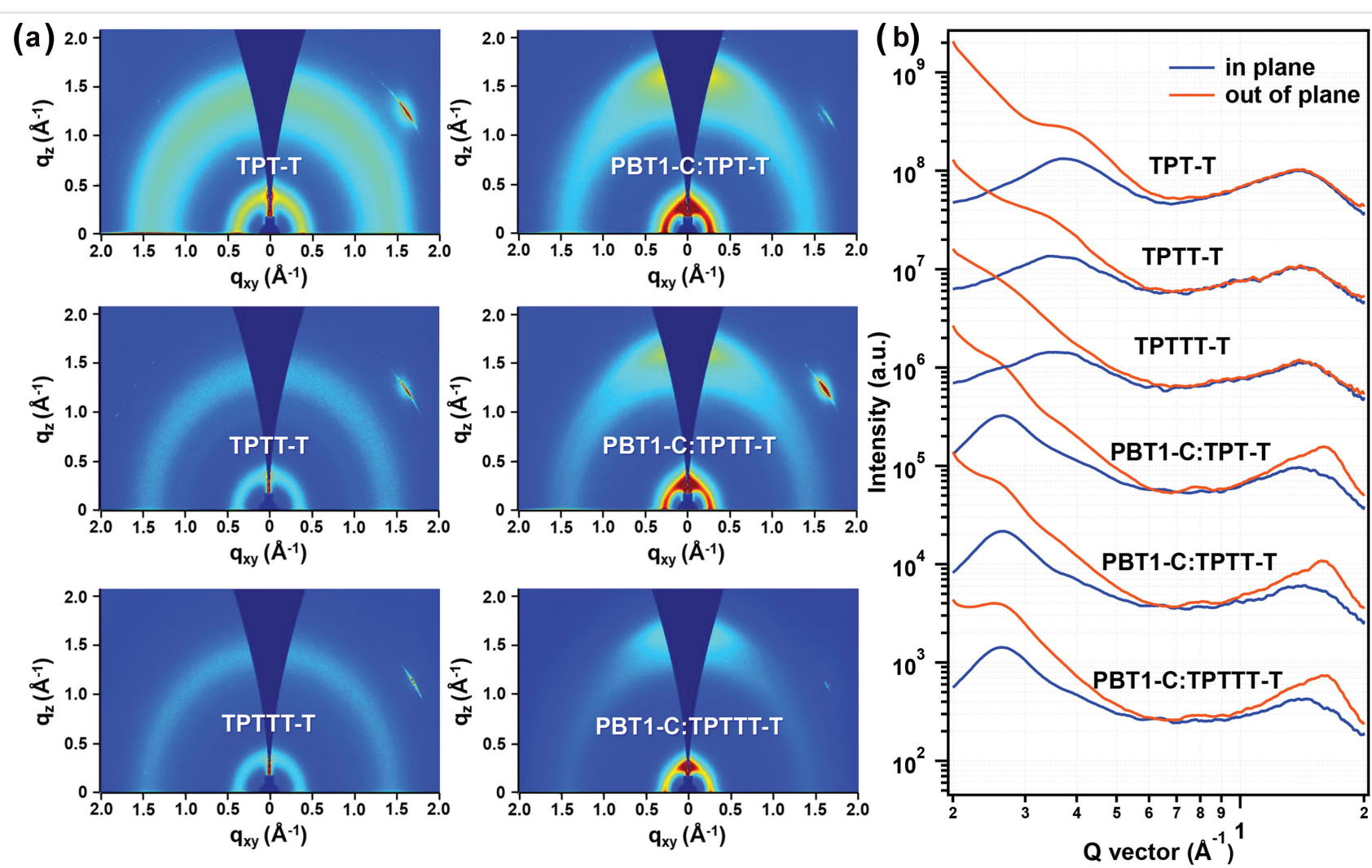

Figure 4 (a) 2D GIWAXS patterns and (b) the corresponding line-cut profiles along the in-plane and out-of-plane directions for the pristine WBG NFAs and their blend films.

the acceptors show weak crystallinity. As shown in Figure S6, the polymer donor PBT1-C presents a sharp (100) lamellar stacking peak in the in-plane (IP) direction together with a well-defined $(010) \pi-\pi$ stacking peak in the out-of-plane (OOP) direction, indicating that PBT1-C mainly prefers the face-on orientation relative to the substrate. After blending PBT1-C with the NFAs, the predominant faceon orientation was still maintained in all the blend films, which is beneficial for vertical charge transport in OSCs. As a result, all the blend films showed high hole mobilities and the electron mobilities of NFAs in the blend films were found to have the same trend in their neat films.

\section{Experimental Section}

\section{Materials}

All reagents and chemicals were purchased from commercial sources and used without further purification unless otherwise noted. The compounds TPT-CHO, ${ }^{66}$ TPTT$\mathrm{CHO}^{30}$ and TPTTT-CHO${ }^{31}$ were synthesized according to the previously reported literature.

\section{Instrumentation}

NMR spectra were measured on a Bruker Avance 300 spectrometer with deuterated chloroform $\left(\mathrm{CDCl}_{3}\right)$ as the solvent and with trimethyl silane (TMS) as the internal reference. UV-visible absorption spectra were measured on a Hitachi (model U-3010) UV-vis spectrophotometer. Mass spectra were recorded on a Bruker Daltonics Biflex III MALDI-TOF analyzer in the MALDI mode. CV measurements were performed under a nitrogen atmosphere at a scan rate of $100 \mathrm{mV} \mathrm{s}{ }^{-1}$ using a Zahner IM6e Electrochemical workstation. A platinum plate coated with sample film was used as a working electrode, a platinum wire was used as a counter electrode, a saturated $\mathrm{Ag} / \mathrm{AgCl}$ electrode was used as a reference electrode, $0.1 \mathrm{M}$ tetra- $n$-butylammonium hexafluorophosphate $\left(\mathrm{Bu}_{4} \mathrm{NPF}_{6}\right)$ in anhydrous acetonitrile solution was employed as a supporting electrolyte and the ferrocene/ferrocenium $\left(\mathrm{Fc} / \mathrm{Fc}^{+}\right)$redox couple was used as an internal standard. The onset oxidation potential of the ferrocene external standard was determined to be $0.43 \mathrm{eV}$. Therefore, the HOMO and LUMO energy levels could be calculated from the following equations: HOMO $=-\left(E_{\text {ox }}{ }^{\text {onset }}+4.37\right) \mathrm{eV}$ and LUMO $=-\left(E_{\text {red }}{ }^{\text {onset }}+4.37\right) \mathrm{eV}$, 
where $E_{\mathrm{ox}}{ }^{\text {onset }}$ and $E_{\mathrm{red}}{ }^{\text {onset }}$ are the onset oxidation potential and onset reduction potential relative to $\mathrm{Ag} / \mathrm{AgCl}$, respectively. AFM measurements were performed using a Dimension Icon AFM (Bruker) in the tapping mode. GIWAXS measurements were performed at the PLS-II 9A U-SAXS beamline of the Pohang Accelerator Laboratory in Korea.

\section{Organic Solar Cell Fabrication and Characterization}

OSCs with an inverted device structure of ITO/ZnO/ PBT1-C:NFA/ $\mathrm{MoO}_{3} / \mathrm{Ag}$ were fabricated. The ITO-coated glass substrates were successively cleaned by ultrasonic treatment with a detergent, deionized water, acetone, and isopropyl alcohol for 20 minutes, respectively. After drying for one night, the $\mathrm{ZnO}$ precursor solution was spin-coated at $4,000 \mathrm{rpm}$ and the $\mathrm{ZnO}$ layer was generated by thermal treatments at $200{ }^{\circ} \mathrm{C}$ for 15 minutes in an ambient atmosphere. In a $\mathrm{N}_{2}$-filled glovebox, the active layers were spin-coated from a solution of PBT1-C: TPT-T, PBT1-C:TPTT-T, or PBT1-C:TPTTT-T with a D/A weight ratio of $1: 1$ in chlorobenzene, and all of them were stirred overnight prior to cast. The average thickness of the active layer was controlled at $90-120 \mathrm{~nm}$. The active layers were allowed to be annealed at $120{ }^{\circ} \mathrm{C}$ for 10 minutes for PBT1-C:TPT-T and at $140{ }^{\circ} \mathrm{C}$ for 10 minutes for PBT1-C:TPTT-T and PBT1-C:TPTTT-T in a $\mathrm{N}_{2}$-filled glovebox. The $\mathrm{MoO}_{3}(3 \mathrm{~nm})$ and $\mathrm{Ag}(90 \mathrm{~nm})$ were deposited by sequential thermal evaporation. Current density-voltage $(J-V)$ characteristics were measured using a Keithley 2400 Source Measure unit. The currents were measured under a $100 \mathrm{~mW} / \mathrm{cm}^{2}$ simulated 1.5 Global (AM 1.5 G) solar simulator (Enli Technology Co., Ltd, SS-F5-3A). The light intensity was calibrated by a standard Si solar cell (SRC-2020, Enli Technology Co., Ltd). EQE spectra were measured on a solar-cell spectralresponse measurement system (QE-R, Enlitech).

\section{Space-Charge Limited Current Measurement}

The charge transport properties were evaluated via a SCLC method. The electron-only devices were fabricated with a structure of ITO/ZnO/PBT1-C:NFA/ZrAcac/Al, and the hole-only devices were fabricated with a structure of ITO/PEDOT:PSS/PBT1-C:NFA/MoO $/$ Ag. The $J-V$ curves of the devices were fitted by using the Mott-Gurney equation: $J=9 \varepsilon_{\mathrm{o}} \varepsilon_{\mathrm{r}} \mu V^{2} / 8 L^{3}$, where $J$ is the current density, $\varepsilon_{0}$ is the permittivity of free space, $\varepsilon_{\mathrm{r}}$ is the permittivity of the active layer, $\mu$ is the hole mobility or electron mobility, $V$ is the internal voltage of the device $\left(V=V_{\mathrm{appl}}-V_{\mathrm{bi}}\right)$ (where $V_{\mathrm{app}}$ is the applied voltage and $V_{\mathrm{bi}}$ is the offset voltage), and $L$ is the film thickness of the active layer. The electron/hole mobility could be calculated from the $J^{0.5}-V$ curve.

\section{Conclusions}

In summary, three A-D-A-type WBG NFAs were developed with different fused central cores by varying $\pi$ conjugation extension and molecular symmetry (from symmetric to asymmetric). As the fused central core was gradually extended, asymmetric TPTTT-T displayed more red-shifted absorption, lower optical bandgaps, upshifted LUMO energy levels, and enhanced electron mobilities compared with symmetric TPT-T. As a consequence, the optimized OSCs based on PBT1-C:TPTTT-T yielded a PCE of $7.42 \%$, which was higher than those of PBT1-C:TPTT-T (6.93\%) and PBT1-C:TPT-T (6.11\%) based devices. Our results may suggest a useful design tip for efficient asymmetric NFAs for OSCs.

\section{Funding Information}

This work was financially supported by the National Natural Science Foundation of China (NSFC: 21734001, 51825301, 21674007). H.Y.W. is grateful for the financial support from the National Research Foundation (NRF) of Korea (NRF2016M1A2A2940911, 2019R1A6A1A11044070).

\section{Supporting Information}

Supporting information for this article is available online at https://doi.org/10.1055/s-0040-1709999.

\section{References}

(1) Li, G.; Zhu, R.; Yang, Y. Nat. Photonics 2012, 6, 153.

(2) Heeger, A. J. Adv. Mater. 2014, 26, 10.

(3) Cai, Y.; Huo, L.; Sun, Y. Adv. Mater. 2017, 29, 1605437.

(4) Fu, H.; Wang, Z.; Sun, Y. Solar RRL 2018, 2, 1700158.

(5) Xie, Y.; Huo, L.; Fan, B.; Fu, H.; Cai, Y.; Zhang, L.; Li, Z.; Wang, Y.; Ma, W.; Chen, Y.; Sun, Y. Adv. Funct. Mater. 2018, 28, 1800627.

(6) Fu, H.; Wang, Z.; Sun, Y. Angew. Chem. Int. Ed. 2019, 58, 4442.

(7) Xia, T.; Cai, Y.; Fu, H.; Sun, Y. Sci. China Chem. 2019, 62, 662.

(8) Günes, S.; Neugebauer, H.; Sariciftci, N. S. Chem. Rev. 2007, 107, 1324.

(9) Huo, L.; Liu, T.; Sun, X.; Cai, Y.; Heeger, A. J.; Sun, Y. Adv. Mater. 2015, 27, 2938.

(10) Huo, L.; Liu, T.; Fan, B.; Zhao, Z.; Sun, X.; Wei, D.; Yu, M.; Liu, Y.; Sun, Y. Adv. Mater. 2015, 27, 6969.

(11) Yao, H.; Ye, L.; Zhang, H.; Li, S.; Zhang, S.; Hou, J. Chem. Rev. 2016, 116, 7397.

(12) Xie, Y.; Yang, F.; Li, Y.; Uddin, M. A.; Bi, P.; Fan, B.; Cai, Y.; Hao, X.; Woo, H. Y.; Li, W.; Liu, F.; Sun, Y. Adv. Mater. 2018, 30, 1803045.

(13) Liu, T.; Troisi, A. Adv. Mater. 2013, 25, 1038.

(14) Yan, C.; Barlow, S.; Wang, Z.; Yan, H.; Jen, A. K. Y.; Marder, S. R.; Zhan, X. Nat. Rev. Mater. 2018, 3, 18003.

(15) Lin, Y.; Zhan, X. Mater. Horiz. 2014, 1, 470.

(16) Cheng, P.; Li, G.; Zhan, X.; Yang, Y. Nat. Photonics 2018, 12, 131.

(17) Lin, Y.; Wang, J.; Zhang, Z-G.; Bai, H.; Li, Y.; Zhu, D.; Zhan, X. Adv. Mater. 2015, 27, 1170. 
(18) Lin, Y.; He, Q.; Zhao, F.; Huo, L.; Mai,J.; Lu, X.; Su, C-J.; Li, T.; Wang,J.; Zhu, J.; Sun, Y.; Wang, C.; Zhan, X.J. Am. Chem. Soc. 2016, 138, 2973.

(19) Sun, D.; Meng, D.; Cai, Y.; Fan, B.; Li, Y.; Jiang, W.; Huo, L.; Sun, Y.; Wang, Z. J. Am. Chem. Soc. 2015, 137, 11156.

(20) Meng, D.; Sun, D.; Zhong, C.; Liu, T.; Fan, B.; Huo, L.; Li, Y.; Jiang, W.; Choi, H.; Kim, T.; Kim, J. Y.; Sun, Y.; Wang, Z.; Heeger, A. J. J. Am. Chem. Soc. 2016, 138, 375.

(21) Meng, D.; Fu, H.; Xiao, C.; Meng, X.; Winands, T.; Ma, W.; Wei, W.; Fan, B.; Huo, L.; Doltsinis, N. L.; Li, Y.; Sun, Y.; Wang, Z. J. Am. Chem. Soc. 2016, 138, 10184.

(22) Liang, N.; Zhu, X.; Zheng, Z.; Meng, D.; Liu, G.; Zhang, J.; Li, S.; Li, Y.; Hou, J.; Hu, B.; Wang, Z. Chem. Mater. 2019, 31, 3636.

(23) Lin, H.; Chen, S.; Li, Z.; Lai, J. Y. L.; Yang, G.; McAfee, T.; Jiang, K.; Li, Y.; Liu, Y.; Hu, H.; Zhao, J.; Ma, W.; Ade, H.; Yan, H. Adv. Mater. 2015, 27, 7299.

(24) Li, S.; Ye, L.; Zhao, W.; Zhang, S.; Mukherjee, S.; Ade, H.; Hou, J. Adv. Mater. 2016, 28, 9423.

(25) Zhao, W.; Li, S.; Yao, H.; Zhang, S.; Zhang, Y.; Yang, B.; Hou, J. J. Am. Chem. Soc. 2017, 139, 7148.

(26) Yao, H.; Chen, Y.; Qin, Y.; Yu, R.; Cui, Y.; Yang, B.; Li, S.; Zhang, K.; Hou, J. Adv. Mater. 2016, 28, 8283.

(27) Cui, Y.; Yao, H.; Hong, L.; Zhang, T.; Xu, Y.; Xian, K.; Gao, B.; Qin, J.; Zhang, J.; Wei, Z.; Hou, J. Adv. Mater. 2019, 31, 1808356.

(28) Zhang, H.; Yao, H.; Hou, J.; Zhu, J.; Zhang, J.; Li, W.; Yu, R.; Gao, B.; Zhang, S.; Hou, J. Adv. Mater. 2018, 30, 1800613.

(29) Xie, D.; Liu, T.; Gao, W.; Zhong, C.; Huo, L.; Luo, Z.; Wu, K.; Xiong, W.; Liu, F.; Sun, Y.; Yang, C. Solar RRL 2017, 1, 1700044.

(30) Li, C.; Xie, Y.; Fan, B.; Han, G.; Yi, Y.; Sun, Y.J. Mater. Chem. C 2018, 6, 4873.

(31) Song, J.; Li, C.; Ye, L.; Koh, C.; Cai, Y.; Wei, D.; Woo, H. Y.; Sun, Y.J. Mater. Chem. A 2018, 6, 18847.

(32) Li, C.; Song, J.; Ye, L.; Koh, C.; Weng, K.; Fu, H.; Cai, Y.; Xie, Y.; Wei, D.; Woo, H. Y.; Sun, Y. Solar RRL 2019, 3, 1800246.

(33) Li, C.; Song, J.; Cai, Y.; Han, G.; Zheng, W.; Yi, Y.; Ryu, H. S.; Woo, H. Y.; Sun, Y. J. Energy Chem. 2020, 40, 144.

(34) Li, C.; Xia, T.; Song, J.; Fu, H.; Ryu, H. S.; Weng, K.; Ye, L.; Woo, H. Y.; Sun, Y. J. Mater. Chem. A 2019, 7, 1435.

(35) Ye, L.; Xie, Y.; Xiao, Y.; Song, J.; Li, C.; Fu, H.; Weng, K.; Lu, X.; Tan, S.; Sun, Y. J. Mater. Chem. A 2019, 7, 8055.

(36) Xie, R.; Ying, L.; Liao, H.; Chen, Z.; Huang, F.; Cao, Y. Front. Chem. 2018, 6, 303.

(37) Wang, J-L.; Liu, K-K.; Hong, L.; Ge, G-Y.; Zhang, C.; Hou, J. ACS Energy Lett. 2018, 3, 2967.

(38) Chang, S-L.; Cao, F-Y.; Huang, W-C.; Huang, P-K.; Huang, K-H.; Hsu, C-S.; Cheng, Y-J. ACS Energy Lett. 2018, 3, 1722.

(39) Liu, Y.; Zuo, L.; Shi, X.; Jen, A. K. Y.; Ginger, D. S. ACS Energy Lett. 2018, 3, 2396.

(40) Li, Y.; Lin, J-D.; Che, X.; Qu, Y.; Liu, F.; Liao, L-S.; Forrest, S. R.J. Am. Chem. Soc. 2017, 139, 17114.

(41) Zhang, G.; Zhao, J.; Chow, P. C. Y.; Jiang, K.; Zhang, J.; Zhu, Z.; Zhang, J.; Huang, F.; Yan, H. Chem. Rev. 2018, 118, 3447.

(42) Zhai, W.; Tang, A.; Xiao, B.; Wang, X.; Chen, F.; Zhou, E. Sci. Bull. 2018, 63, 845.

(43) Huang, C.; Liao, X.; Gao, K.; Zuo, L.; Lin, F.; Shi, X.; Li, C-Z.; Liu, H.; Li, X.; Liu, F.; Chen, Y.; Chen, H.; Jen, A. K. Y.Chem. Mater. 2018, 30, 5429.

(44) Shi, X.; Zuo, L.; Jo, S. B.; Gao, K.; Lin, F.; Liu, F.; Jen, A. K. Y. Chem. Mater. 2017, 29, 8369.
(45) Shi, X.; Liao, X.; Gao, K.; Zuo, L.; Chen, J.; Zhao, J.; Liu, F.; Chen, Y.; Jen, A. K. Y. Adv. Funct. Mater. 2018, 28, 1802324.

(46) Sun, J.; Ma, X.; Zhang, Z.; Yu, J.; Zhou, J.; Yin, X.; Yang, L.; Geng, R.; Zhu, R.; Zhang, F.; Tang, W. Adv. Mater. 2018, 30, 1707150.

(47) Wang, J.; Wang, W.; Wang, X.; Wu, Y.; Zhang, Q.; Yan, C.; Ma, W.; You, W.; Zhan, X. Adv. Mater. 2017, 29, 1702125.

(48) Zhao, F.; Dai, S.; Wu, Y.; Zhang, Q.; Wang, J.; Jiang, L.; Ling, Q.; Wei, Z.; Ma, W.; You, W.; Wang, C.; Zhan, X. Adv. Mater. 2017, 29, 1700144.

(49) Li, Y.; Zhong, L.; Gautam, B.; Bin, H-J.; Lin, J-D.; Wu, F-P.; Zhang, Z.; Jiang, Z-Q.; Zhang, Z-G.; Gundogdu, K.; Li, Y.; Liao, L-S. Energy Environ. Sci. 2017, 10, 1610.

(50) Li, W.; Ye, L.; Li, S.; Yao, H.; Ade, H.; Hou, J. Adv. Mater. 2018, 30, 1707170 .

(51) Kan, B.; Zhang, J.; Liu, F.; Wan, X.; Li, C.; Ke, X.; Wang, Y.; Feng, H.; Zhang, Y.; Long, G.; Friend, R. H.; Bakulin, A. A.; Chen, Y. Adv. Mater. 2018, 30, 1704904.

(52) Zhang, Y.; Kan, B.; Sun, Y.; Wang, Y.; Xia, R.; Ke, X.; Yi, Y-Q-Q.; Li, C.; Yip, H-L.; Wan, X.; Cao, Y.; Chen, Y. Adv. Mater. 2018, 30, 1707508.

(53) Zuo, L.; Yu, J.; Shi, X.; Lin, F.; Tang, W.; Jen, A. K. Y. Adv. Mater. 2017, 29, 1702547.

(54) Shi, X.; Chen, J.; Gao, K.; Zuo, L.; Yao, Z.; Liu, F.; Tang, J.; Jen, A. K. Y. Adv. Energy Mater. 2018, 8, 1702831.

(55) Zuo, L.; Shi, X.; Jo, S. B.; Liu, Y.; Lin, F.; Jen, A. K. Y. Adv. Mater. 2018, 30, 1706816.

(56) Yao, H.; Cui, Y.; Yu, R.; Gao, B.; Zhang, H.; Hou, J. Angew. Chem. Int. Ed. 2017, 56, 3045.

(57) He, B.; Yang, B.; Kolaczkowski, M. A.; Anderson, C. A.; Klivansky, L. M.; Chen, T. L.; Brady, M. A.; Liu, Y. ACS Energy Lett. 2018, 3, 1028.

(58) Gong, Y.; Kan, Z.; Xu, W.; Wang, Y.; AlShammari, S. H.; Laquai, F.; Lai, W-Y.; Huang, W. Solar RRL 2018, 2, 1800120.

(59) Xiao, B.; Tang, A.; Zhang, J.; Mahmood, A.; Wei, Z.; Zhou, E. Adv Energy Mater. 2017, 7, 1602269.

(60) Tang, A.; Xiao, B.; Wang, Y.; Gao, F.; Tajima, K.; Bin, H.; Zhang, ZG.; Li, Y.; Wei, Z.; Zhou, E. Adv. Funct. Mater. 2018, 28, 1704507.

(61) Tang, A.; Xiao, B.; Chen, F.; Zhang, J.; Wei, Z.; Zhou, E. Adv. Energy Mater. 2018, 8, 1801582.

(62) Tang, A.; Song, W.; Xiao, B.; Guo, J.; Min, J.; Ge, Z.; Zhang, J.; Wei, Z.; Zhou, E. Chem. Mater. 2019, 31, 3941.

(63) Jia, J.; Zheng, N.; Wang, Z.; Huang, Y.; Duan, C.; Huang, F.; Cao, Y. Sci. China Chem. 2017, 60, 1458.

(64) Wang, P.; Fan, H.; Zhang, C.; Zhu, X. Mater. Chem. Front. 2018, 2, 136.

(65) Li, C.; Fu, H.; Xia, T.; Sun, Y. Adv. Energy Mater. 2019, 9, 1900999.

(66) Ko, E. Y.; Park, G. E.; Lee, J. H.; Kim, H. J.; Lee, D. H.; Ahn, H.; Uddin, M. A.; Woo, H. Y.; Cho, M. J.; Choi, D. H. ACS Appl. Mater. Interfaces 2017, 9, 8838.

(67) Liu, T.; Huo, L.; Chandrabose, S.; Chen, K.; Han, G.; Qi, F.; Meng, X.; Xie, D.; Ma, W.; Yi, Y.; Hodgkiss, J. M.; Liu, F.; Wang, J.; Yang, C.; Sun, Y. Adv. Mater. 2018, 30, 1707353.

(68) Faist, M. A.; Shoaee, S.; Tuladhar, S.; Dibb, G. F. A.; Foster, S.; Gong, W.; Kirchartz, T.; Bradley, D. D. C.; Durrant, J. R.; Nelson, J. Adv. Energy Mater. 2013, 3, 744.

(69) Riedel, I.; Parisi, J.; Dyakonov, V.; Lutsen, L.; Vanderzande, D.; Hummelen, J. C. Adv. Funct. Mater. 2004, 14, 38. 